Tema: Solidificação / Lingotamento

\title{
ESTRATEGIAS CONTRA LA REOXIDACIÓN DEL ACERO LÍQUIDO EN LA COLADA CONTINUA DE PALANQUILLAS CON BUZA CALIBRADA*
}

\begin{abstract}
Jorge Madias $^{1}$ Alberto Moreno ${ }^{2}$

\section{Resumen}

La mayor parte de las máquinas de colada continua de palanquillas que utilizan buzas calibradas y lubricación con aceite se operan con alta velocidad de colada en secuencias largas, obteniendo alta productividad. Muchos de ellos todavía practican la inyección de aluminio en el molde, para desoxidar el acero líquido sin riesgo de obturación de las buzas. También es todavía usual la práctica de "pescar" la nata que se forma en el menisco. Las salpicaduras del chorro de cuchara contribuyen a la formación de chanchos en el repartidor, requiriendo el lanceo con oxígeno. Alternativamente, diversas plantas prefieren utilizar tubo de protección entre cuchara y repartidor y protección con gas inerte del chorro del repartidor al molde en todos los grados de acero colados con buza calibrada. Se hace una comparación entre ambas prácticas desde los puntos de vista de inversión, costo operativo, seguridad, productividad y calidad.
\end{abstract}

Palabras clave: Colada continua; Buza calibrada; Palanquillas; Tochos; Reoxidación; Atrape de escoria; Perforaciones.

\section{STRATEGIES AGAINS REOXIDATION IN BILLET CASTING WITH METERING NOZZLE}

\begin{abstract}
Most billet casters using metering nozzle and oil lubrication operate with large casting speed in long sequences, achieving high productivity. Many of them still use old practices like aluminum injection in the mold, to accomplish deoxidation without the risk of clogging the nozzles. Also, the practice of fishing the scum formed in the meniscus is usual. Splashing from the ladle stream contributes to skulls in the tundish, requiring oxygen lancing. Against this, some companies prefer to use long nozzle and inert gas protection for all steel grades. A comparison is made between both practices from the point of view of investment, operating cost, safety, productivity and billet quality.
\end{abstract}

Key words: Continuous casting; Metering nozzle; Billets; Blooms, Reoxidation; Slag patch; Breakout.

1 Ingeniero Metalúrgico, Director, Metallon, miembro de la ABM, San Nicolas, Buenos Aires, Argentina.

2 Técnico, Consultor, TechnoConsultancy, Villa Constitución, Argentina.

* Contribuição técnica ao 45ํㅗㄴ Seminário de Aciaria - Internacional, 25 a 28 de maio de 2014, 


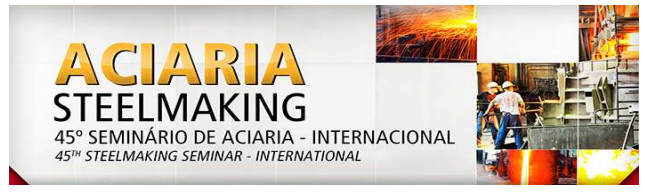

\section{INTRODUCCIÓN}

La colada continua de productos largos a chorro abierto, usando buzas calibradas y lubricación con aceite, es una herramienta eficiente para la obtención de productos largos para la construcción civil con alta productividad y bajo costo, y una calidad aceptable para las aplicaciones apuntadas. Esto incluye barras y alambrón corrugados para refuerzo de hormigón, barras lisas redondas y cuadradas para uso general y perfiles, ángulos y planchuelas pequeños y medianos.

En el marco tecnológico y económico actual no es viable la utilización para estos productos del sistema de colado con buza sumergida y polvo colador, que es típico de las plantas que producen barras y alambrón automotrices y tubos sin costura. Esto tiene relación con la menor productividad inherente a este sistema de colado, limitada por la duración de la buza sumergida y por la aplicación de velocidades de colada inferiores, para el cumplimiento de los requisitos de calidad, y los mayores costos derivados de la utilización de buzas sumergidas (incluyendo también los sistemas de control de caudal barra tapón o con válvula deslizante) y polvos coladores.

La operación clásica de la colada continua a chorro abierto se ha visto potenciada a lo largo de las últimas décadas por la introducción de los hornos cuchara, la implementación de sistemas de cambio automático de buza que permiten aumentar la seguridad ante una emergencia en la máquina; manejar la velocidad de colada en función de las necesidades y cambiar la buza si eventualmente presenta un problema de obturación o de desgaste prematuro; las mejoras en el diseño de los moldes para permitir el colado a alta velocidad, el alto grado de automatización aplicado a las máquinas.

La operación clásica incluye el colado a chorro abierto entre la cuchara y el repartidor, y entre el repartidor y el molde (con la excepción de los aceros de bajo carbono, a lo que se les suele aplicar la protección gaseosa). En algunos casos se continúa con la práctica de adición de aluminio en el molde, que se introdujo en los años 70, cuando todavía no se empleaban masivamente los hornos cuchara, como una forma de completar la desoxidación sin correr el riesgo de la obturación de las buzas calibradas [1]. Otra práctica típica es la "pesca" de la escoria o nata que en esas condiciones se forma en la superficie del molde. El aceite al llegar al punto de inflamación se enciende y despide llamas.

Sin embargo, en las últimas décadas han surgido algunas plantas que emplean para estos aceros el tubo de protección entre la cuchara y el repartidor; utilizan la protección gaseosa entre el repartidor y el molde para todos los aceros producidos, y no exclusivamente para los de bajo carbono; no practican la adición de aluminio en el molde ni la "pesca" de escoria.

Es interesante comparar desde diversos puntos de vista ambas prácticas y cómo influyen en aspectos claves como la seguridad del personal, el costo de inversión y operativo, la productividad y la calidad de los productos (Figura 1).

* Contribuição técnica ao 45 Seminário de Aciaria - Internacional, 25 a 28 de maio de 2014, 

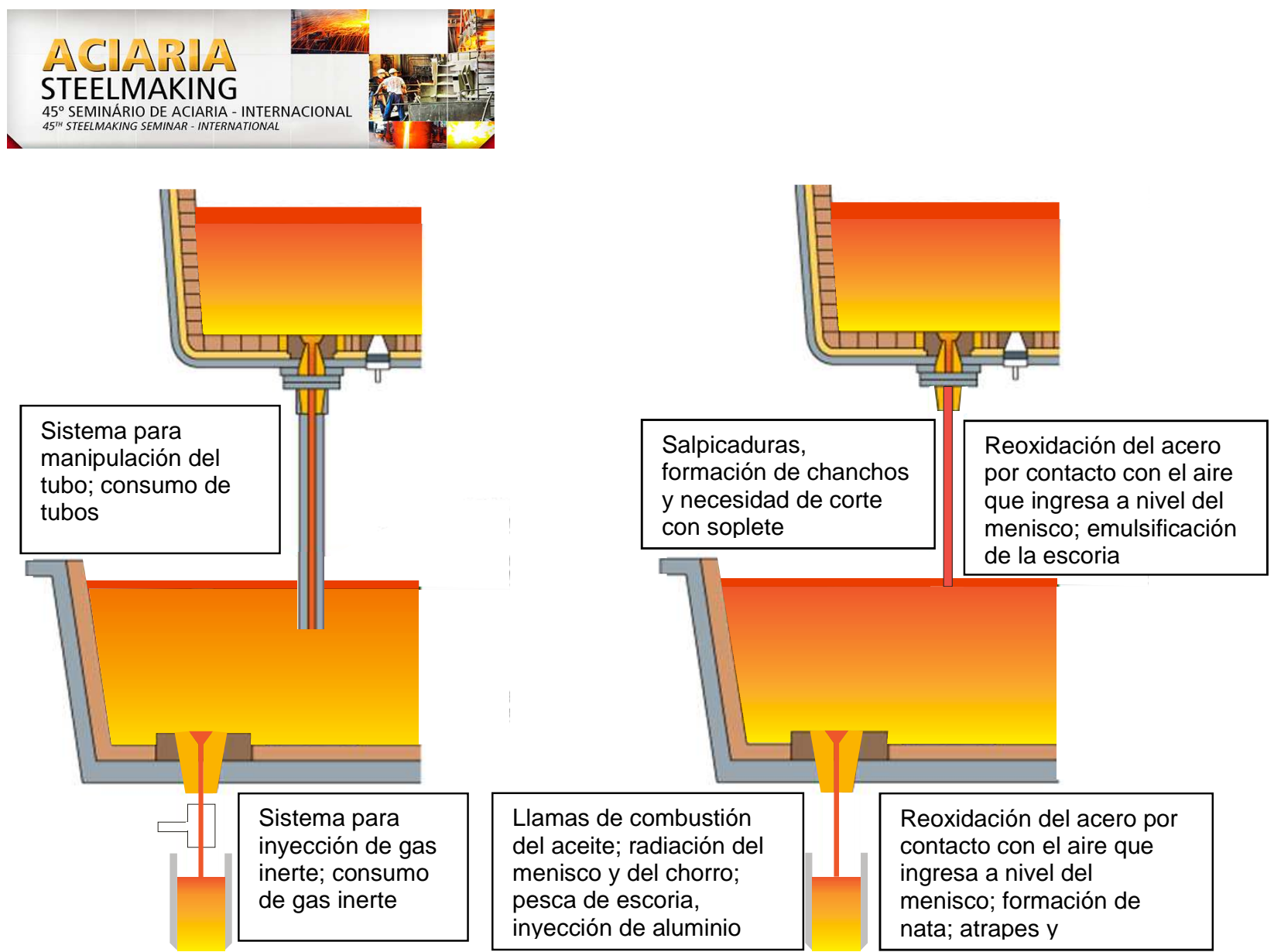

Figura 1. Dos prácticas para el colado con buza calibrada: Izquierda: colado con tubo entre cuchara y repartidor y protección gaseosa entre repartidor y molde; derecha: colado a chorro abierto entre cuchara y repartidor y repartidor y molde.

\section{TRANSFERENCIA CUCHARA - REPARTIDOR}

El colado a chorro abierto simplifica la operación de arranque de la secuencia y los cambios de cuchara, ya que no es necesaria la extracción del tubo de la cuchara vaciada, la inyección de oxígeno para limpiar el interior del tubo para reutilizarlo ni la introducción del tubo en la nueva cuchara. Desde el punto de vista del diseño de la máquina, no es necesario que haya una altura importante entre la cuchara y el repartidor y/o movimiento vertical del repartidor, para facilitar la introducción del tubo. La ausencia del tubo tiene algunas consecuencias negativas. Se producen salpicaduras de gotas de acero, particularmente durante el llenado del repartidor, que afectan el trabajo a la altura del repartidor y eventualmente en la plataforma de colado (Figura 2). La adhesión de algunas de estas salpicaduras en ambas laterales del repartidor, en la zona central, puede llevar a lo largo de la secuencia a la formación de chanchos que requerirán operaciones de oxicorte a la altura la plataforma del repartidor, que presentan riesgos de seguridad [2].

* Contribuição técnica ao 45ํㅗㄴ Seminário de Aciaria - Internacional, 25 a 28 de maio de 2014, Porto Alegre, RS, Brasil. 

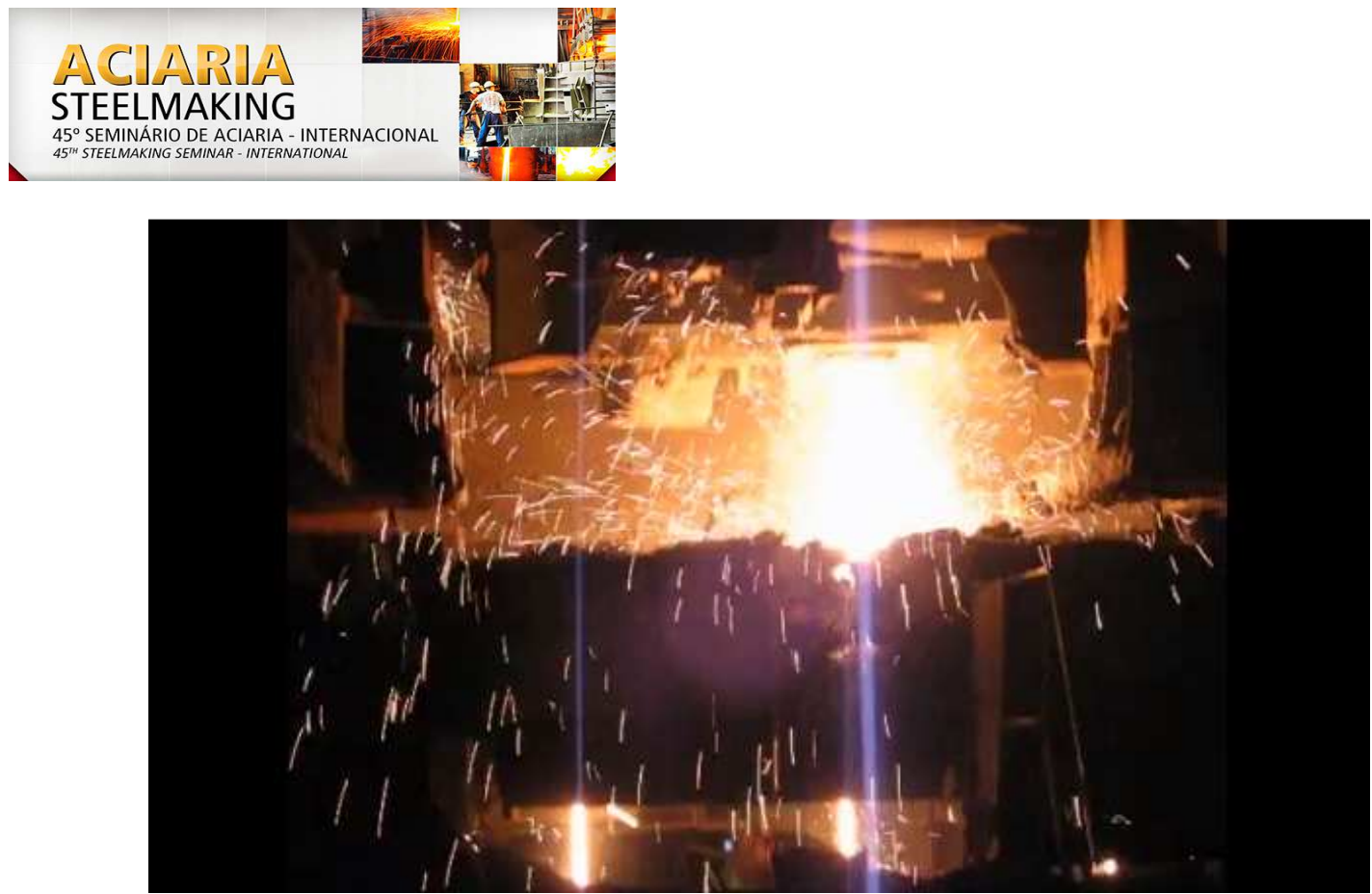

Figura 2. Colado a chorro abierto entre cuchara y repartidor con cuchara de 65 t. Se observa el salpicado de acero durante el llenado del repartidor [3].

El tubo cerámico está construido generalmente en alúmina-grafito, con la técnica de prensado isostático (Figura 3). No requiere precalentamiento. En algunos casos, para prevenir el ataque de la escoria que se acumula a lo largo de la secuencia en el repartidor, tiene un inserto de óxido de circonio. En el extremo superior del tubo, donde se une con la buza externa de la cuchara, la fuerza del chorro descendente genera una depresión importante (efecto Venturi) y puede dar lugar al ingreso de aire. Para evitarlo se utiliza una junta de un material con capacidad de deformación que sella el espacio entre la buza externa y el tubo.

Para mejorar el sellado evitando el ingreso de se puede implementar la inyección de argón, sea mediante un tubo anular perforado alrededor de la cabeza del tubo cerámico, sea que el propio tubo tiene una cabeza porosa para distribuir el argón que ingresa mediante una conexión (Figura 3). Pero esto no es usual para el colador de aceros calmado con silicio y manganeso.

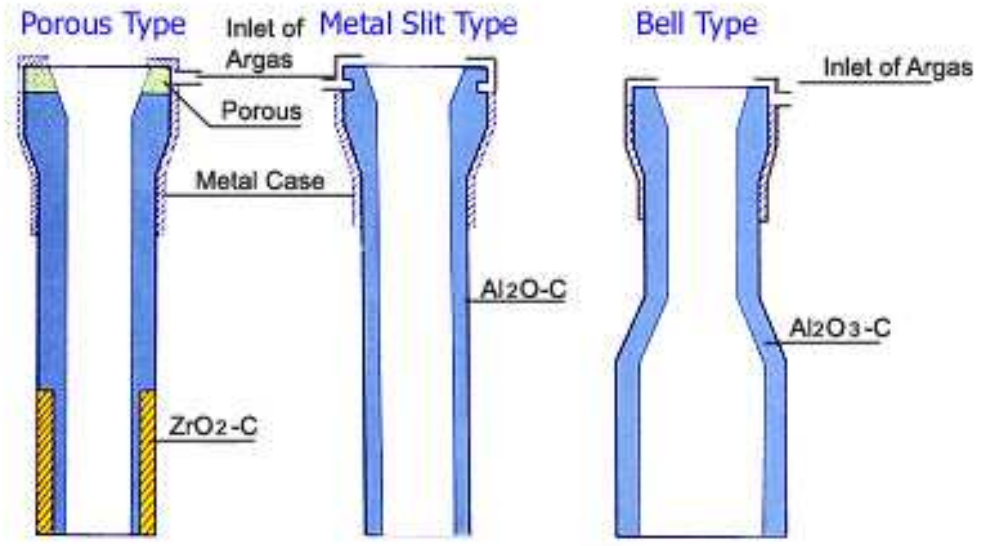

Figura 3. Diversos tipos de tubos de protección. Izquierda: Con cabeza porosa para inyección de argón e inserto de óxido de circonio-carbono para la línea de escoria; centro: Con ranura para inyección de argón. Derecha: Tipo campana, para evitar el retroceso cuando se hace la apertura de la válvula de cuchara con el tubo ya sumergido [4].

El sistema para su colocación consiste en un manipulador ("bicicleta" o "muñeco" que permite posicionarlo y que posee un contrapeso para mantenerlo contra la buza externa. Se opera manualmente. Esto es suficiente en la mayor parte de los casos,

* Contribuição técnica ao 45 Seminário de Aciaria - Internacional, 25 a 28 de maio de 2014, Porto Alegre, RS, Brasil. 


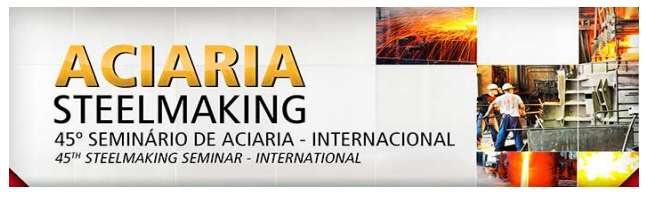

aunque hay también dispositivos que incluyen cilindros hidráulicos que facilitan los esfuerzos (Figura 3); y robots que cumplen esta función y el manejo de la válvula de cuchara.

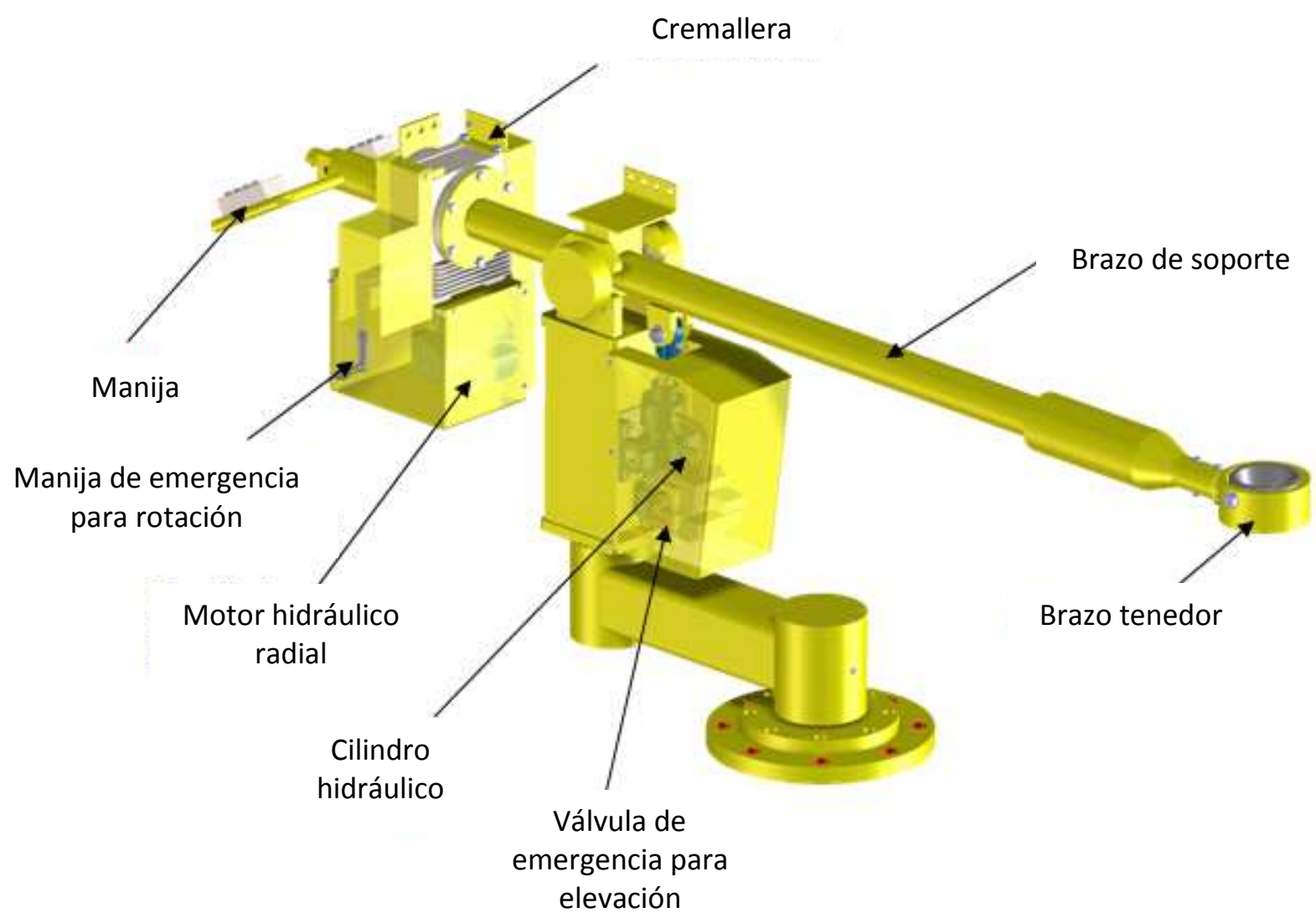

Figura 4. Sistema para la colocación y extracción del tubo de protección [5].

El tubo debe tener una profundidad de inmersión en el acero adecuada, para jugar su rol en lo que hace a la reoxidación y la emulsificación de la escoria. Si la profundidad de inmersión es baja, puede provocar la exposición de acero al contacto con el aire alrededor del tubo yo la emulsificación de la escoria que pudiera estar presente en la superficie, un fenómeno que una vez ocurrido es muy difícil de revertir.

Un ítem importante es lograr la máxima duración del tubo para disminuir su impacto sobre el costo operativo. Una duración típica es de ocho horas. En muchas oportunidades, incide la quiebra del tubo durante la manipulación al extraerlo, limpiarlo o colocarlo, más que el desgaste de las sucesivas operaciones de limpieza con oxígeno, y el ataque de la escoria que se acumular en el repartidor a lo largo de la secuencia.

El tubo de protección, una vez colocado evita el problema de las salpicaduras, la formación de chanchos y la necesidad de corte mencionados anteriormente. En el caso de que no hubiera apertura de la cuchara cuando se desplaza la válvula, lo usual es extraer el tubo, proceder a la apertura con lanza y esperar al llenado del repartidor para volverlo a colocar; en la recolocación no se cierra totalmente la placa. Si se da una situación de obturación de buza de cuchara, que no debería suceder con un buen proceso en el horno cuchara, el tubo se extrae, se limpia con lanza la buza de cuchara y se vuelve a colocar.

* Contribuição técnica ao 45 Seminário de Aciaria - Internacional, 25 a 28 de maio de 2014, 


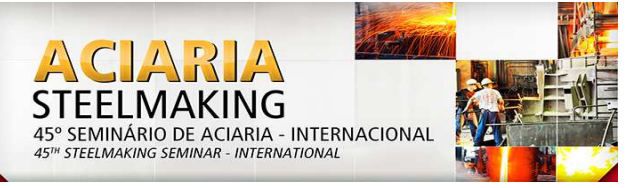

\section{TRANSFERENCIA REPARTIDOR - MOLDE}

Si bien la altura del chorro y su diámetro son menores, los fenómenos que suceden a nivel del menisco son similares a los que suceden con el chorro de la cuchara. El chorro abierto también simplifica la operación de arranque, ya que no es necesario colocar el "tambucho" y abrir el paso al gas inerte una vez abiertas las buzas e iniciada la colada. Aquí, al ser la operación continua, no inciden los cambios de cuchara.

La protección gaseosa consiste en un sistema de cañerías con válvulas reguladoras que proporcionan un caudal uniforme en todas las líneas para el paso del gas inerte, sea argón o nitrógeno, y una cobertura que permite mantener una atmósfera inerte sobre el menisco y alrededor del chorro. Esta cobertura se logra de diferentes maneras: mediante un fuelle o mediante un "tambucho", ver Figura 5. Un consumo específico típico de gas está en el orden de $0,2 \mathrm{Nm}^{3} / \mathrm{t}$ de acero.

Debe tenerse en cuenta en el diseño del sistema que el molde oscila, que el sistema de cambio automático de buza no debe recalentarse, que debe haber espacio para poder pasar el canal en caso de una emergencia que no pueda ser resuelta con una buza ciega (particularmente en arranques). Y que si bien una desoxidación adecuada en el horno cuchara y el correcto funcionamiento de las protecciones durante la transferencia hace que no sean necesarias la inyección de aluminio en el molde y la "pesca" de escoria, pueden darse situaciones en que sea necesario acceder a la buza o al menisco.

Una buena parte de los sistemas en uso ha sido desarrollada "in house", generalmente con participación de los propios operadores de las máquinas, pero también hay en el mercado empresas que ofrecen la instalación. En la Figura 6 se presenta un detalle de un sistema con fuelle, que además de la posición de trabajo tiene una posición para la inspección de la buza calibrada y otra para la inspección del molde.

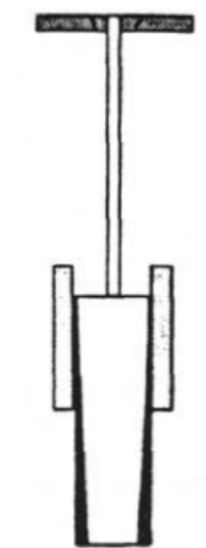

Chorro

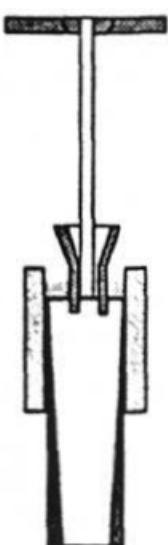

Embudo y polvo

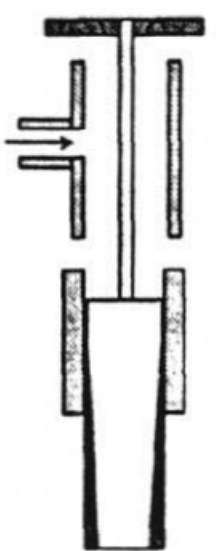

Tambucho

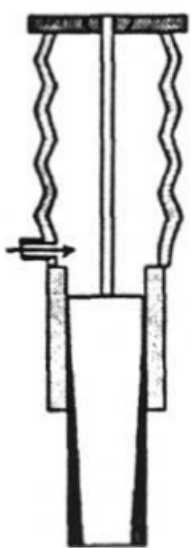

Fuelle

Figura 5. Diversos sistemas para la protección de la transferencia de acero del repartidor al molde mediante gas inerte, en el colado con buza calibrada [6].

* Contribuição técnica ao 45 Seminário de Aciaria - Internacional, 25 a 28 de maio de 2014, 

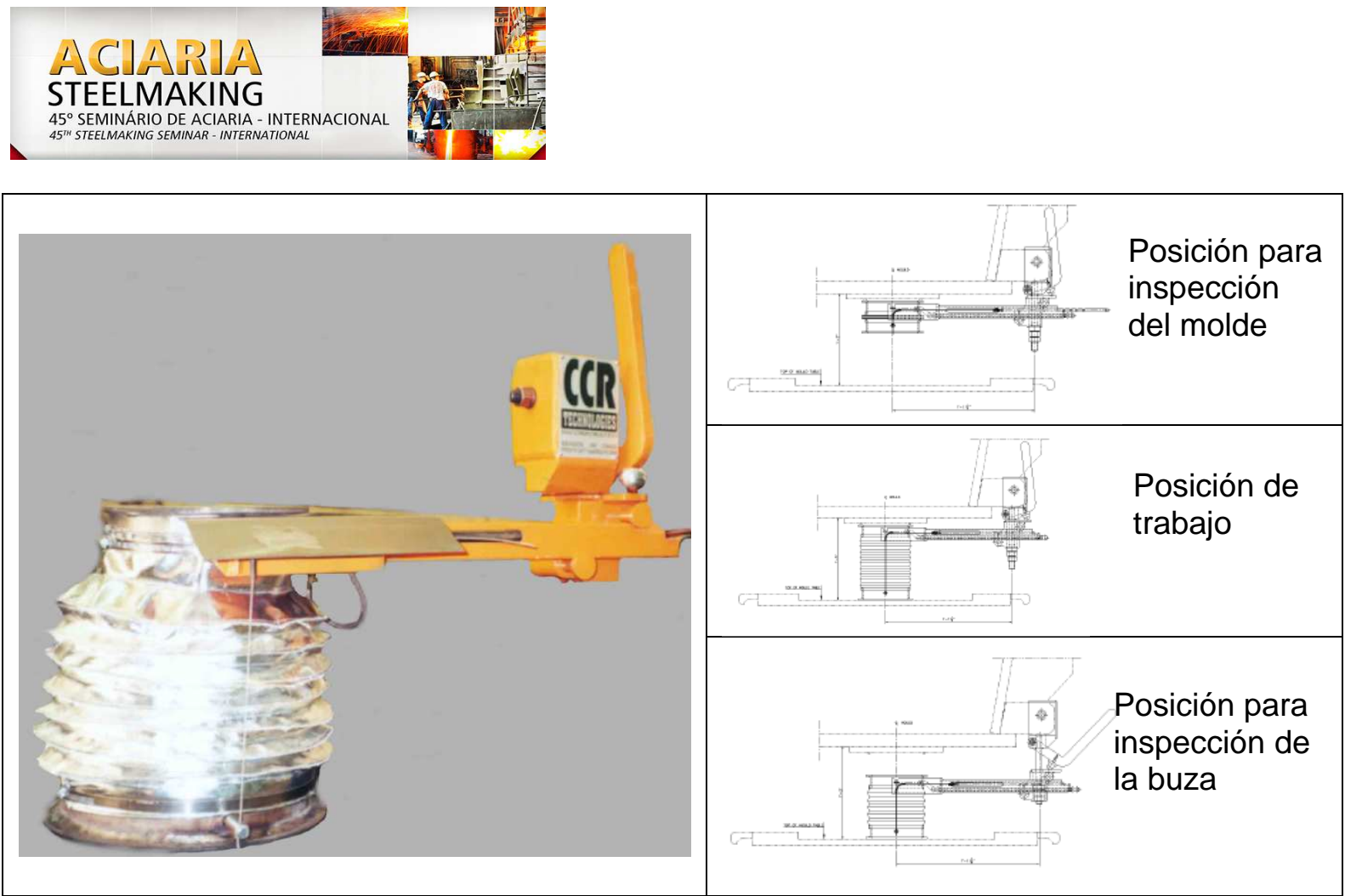

Figura 6. Protección de chorro con gas inerte mediante fuelle. Izquierda: Aspecto del fuelle y dispositivo para su manipulación. Derecha: posición del fuelle para operación, inspección de buza e inspección del molde [7].

Es posible controlar en forma directa la efectividad de la protección mediante la utilización de un analizador portátil de gases. Es recomendable que el tenor de oxígeno en la zona cercana al menisco sea menor de $0,5 \%$ de oxígeno.

El uso de argón o nitrógeno depende de los costos relativos, la disponibilidad y del cumplimiento de la especificación de nitrógeno. Debe tenerse en cuenta que para los aceros de bajo carbono, con alta actividad de oxígeno, la absorción de nitrógeno en la transferencia repartidor - molde es baja.

\section{REOXIDACIÓN, MACROINCLUSIONES Y FORMACIÓN DE NATA}

Los estudios de Maddever, McLean y otros [8-9], permitieron entender el mecanismo involucrado en la reoxidación del acero por contacto con el aire. En la zona alrededor del chorro se produce un anillo que periódicamente da lugar a la incorporación de burbujas de aire al acero, que siguen una trayectoria dentro del mismo, hasta volver a emerger (Figura 7). La formación de estas burbujas implica una gran superficie para la reacción entre el oxígeno del aire y el acero líquido. Esto implica que aunque el chorro sea uniforme, ofreciendo poca superficie a la acción del aire, la reoxidación tiene lugar igualmente, particularmente en la zona por debajo del chorro. Se ha calculado que la cantidad de oxígeno introducida por el chorro en el menisco es 100 veces mayor que la absorbida por transferencia de masa en la superficie chorro -aire, para um chorro uniforme. 

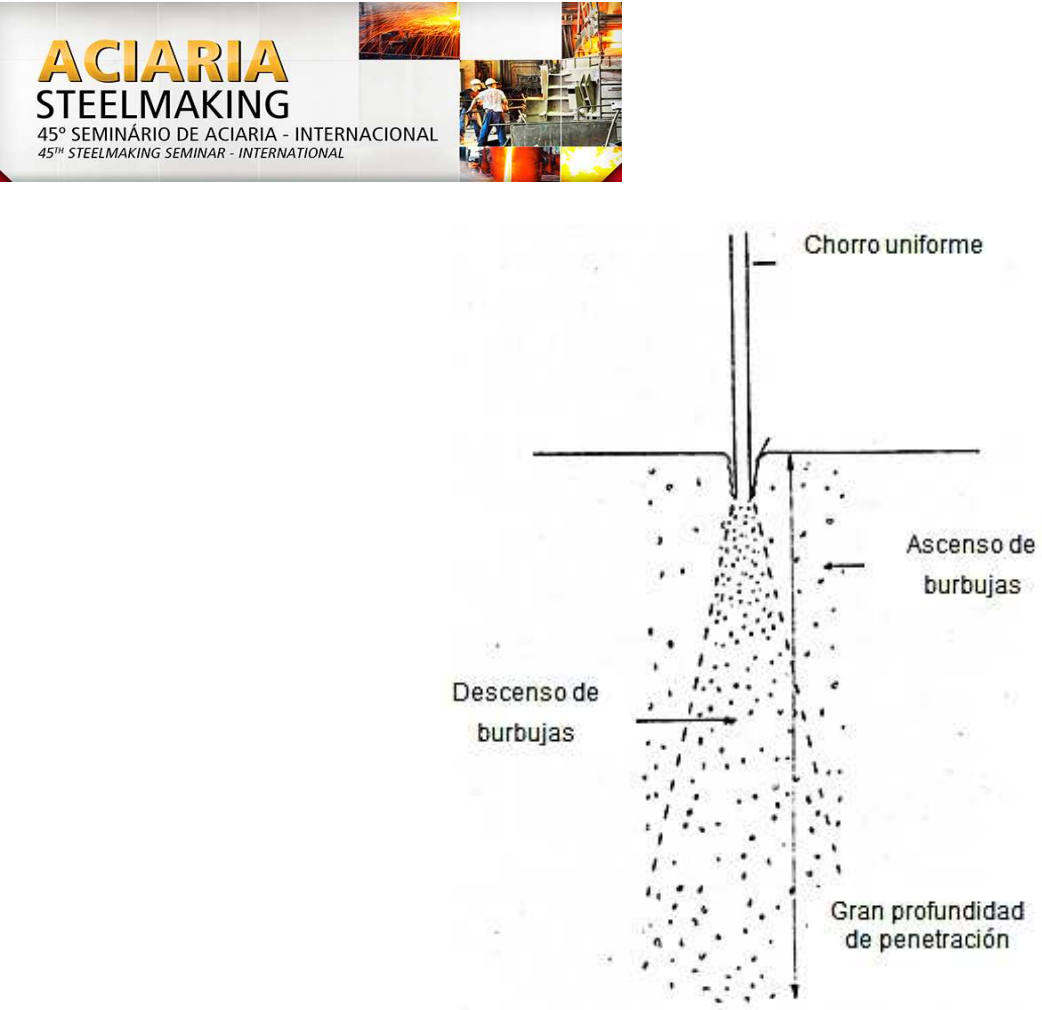

Figura 7. Mecanismo para la reoxidación del acero por contacto con el aire en el colado a chorro abierto, con chorro uniforme [9].

Los aceros para el colado con buza calibrada son calmados con silicio, manganeso y pequeñas cantidades de aluminio, sea que se adicione aluminio ex profeso, como sucede en los aceros de bajo carbono, sea que intervenga el aluminio introducido con las ferroaleaciones. Los elementos que se oxidarán más rápidamente en contacto con el aire son el aluminio que puede estar presente en pequeñas cantidades, el silicio y el manganeso, que están presentes en concentraciones mucho mayores. El resultado es que se forman macroinclusiones de reoxidación ricas en $\mathrm{SiO}_{2}$ y $\mathrm{MnO}$ pero con un contenido menor de $\mathrm{Al}_{2} \mathrm{O}_{3}$ que las microinclusiones de desoxidación (Figura 8, izquierda).
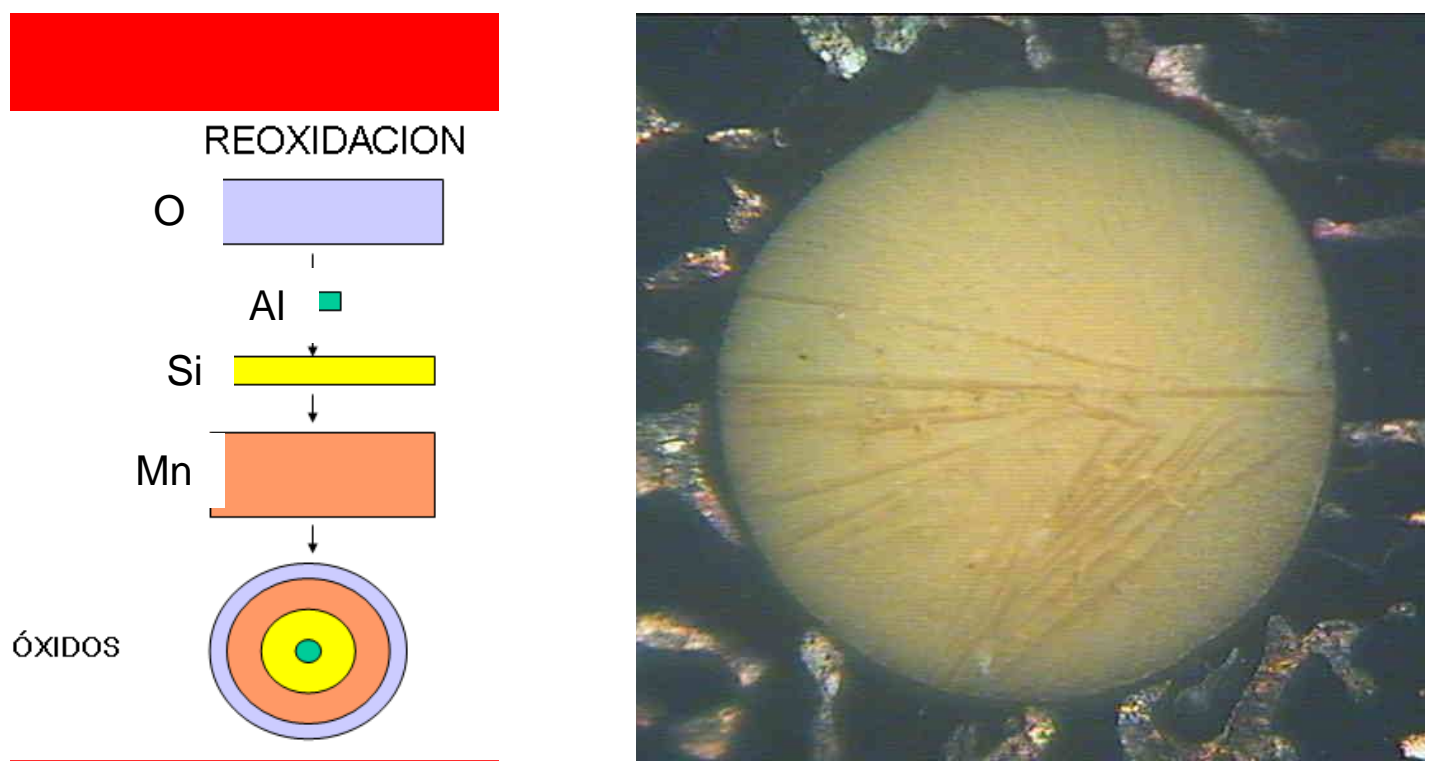

Figura 8. Macroinclusiones de reoxidación en aceros calmados con $\mathrm{Si}, \mathrm{Mn}$ y pequeñas cantidades de Al. Izquierda: Interpretación de la composición química de las macroinclusiones de reoxidación en aceros calmados con silicio, manganeso y pequeña cantidad de aluminio [10]. Derecha: Macroinclusión en palanquilla. Matriz vítrea de silicato de manganeso y aluminio, y agujas de rodonita precipitadas. Observación en microscopio óptico, bajo luz polarizada.

* Contribuição técnica ao 45 Seminário de Aciaria - Internacional, 25 a 28 de maio de 2014, Porto Alegre, RS, Brasil. 
Debe tenerse en cuenta que el contacto con el aire favorece también la incorporación de nitrógeno, que puede ser perjudicial desde el punto de vista de la formación de sopladuras y en algunos aceros desde el punto de vista del envejecimiento por la deformación durante la trefilación. Esto es importante sobre todo en aceros con bajo tenor de azufre y oxígeno disuelto.

Las macroinclusiones pueden dar lugar a la formación de nata o escoria en el molde, por flotación y acumulación en el menisco (Figura 9).

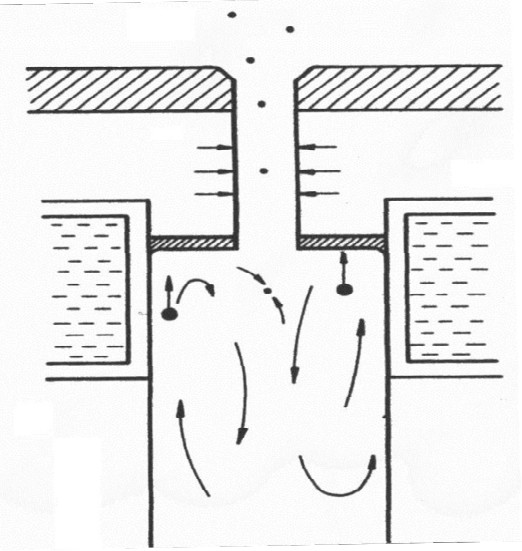

Figura 9. Mecanismo de formación de escoria de molde (nata) por acumulación de macroinclusiones de reoxidación.

Esta nata que sobrenada en el molde puede dar lugar a atrapes superficiales y perforaciones de línea, en particular cuando en ella precipita una fase sólida, incrementado la viscosidad. Hay dos situaciones típicas en que esto sucede: cuando precipita sílice o cuando precipita alúmina. La precipitación de sílice es función del equilibrio entre el acero, con su contenido de silicio y manganeso, y la escoria, a una temperatura dada (Figura 10). Una forma simplificada de expresar la necesidad de evitar esta precipitación es la limitación de la relación $\mathrm{Mn} / \mathrm{Si}$ a un mínimo de 3. La precipitación de alúmina es típica de cuando se utiliza la inyección de aluminio (Figura 11).

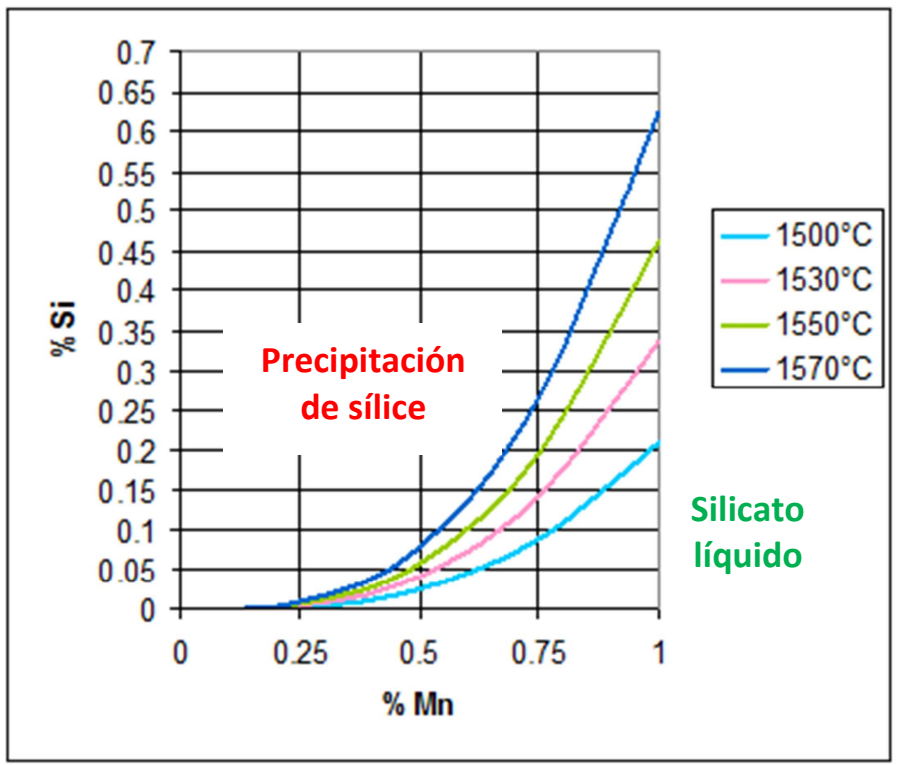

Figura 10. Equilibrio entre un acero de bajo carbono calmado con silicio y manganeso y la escoria sobrenadante en el molde, a diversas temperaturas. Por encima de las curvas sucede la precipitación de sílice [11].

* Contribuição técnica ao 45 Seminário de Aciaria - Internacional, 25 a 28 de maio de 2014, Porto Alegre, RS, Brasil. 


\section{ACEITE}

Cabe mencionar que el uso efectivo de la protección del acero en la transferencia del repartidor al molde modifica el comportamiento del aceite. La generación de llama típica de la combustión del aceite con el aire presente en la parte superior del molde, genera por una parte un problema de ambiente laboral inadecuado y en casos de adición exagerada puede llevar incluso al recalentamiento del sistema de cambio automático de buza. Si no hay oxígeno, la combustión se minimiza, las llamas se reducen a un mínimo y es probable que se requiera menos aceite para lograr el mismo efecto lubricante.

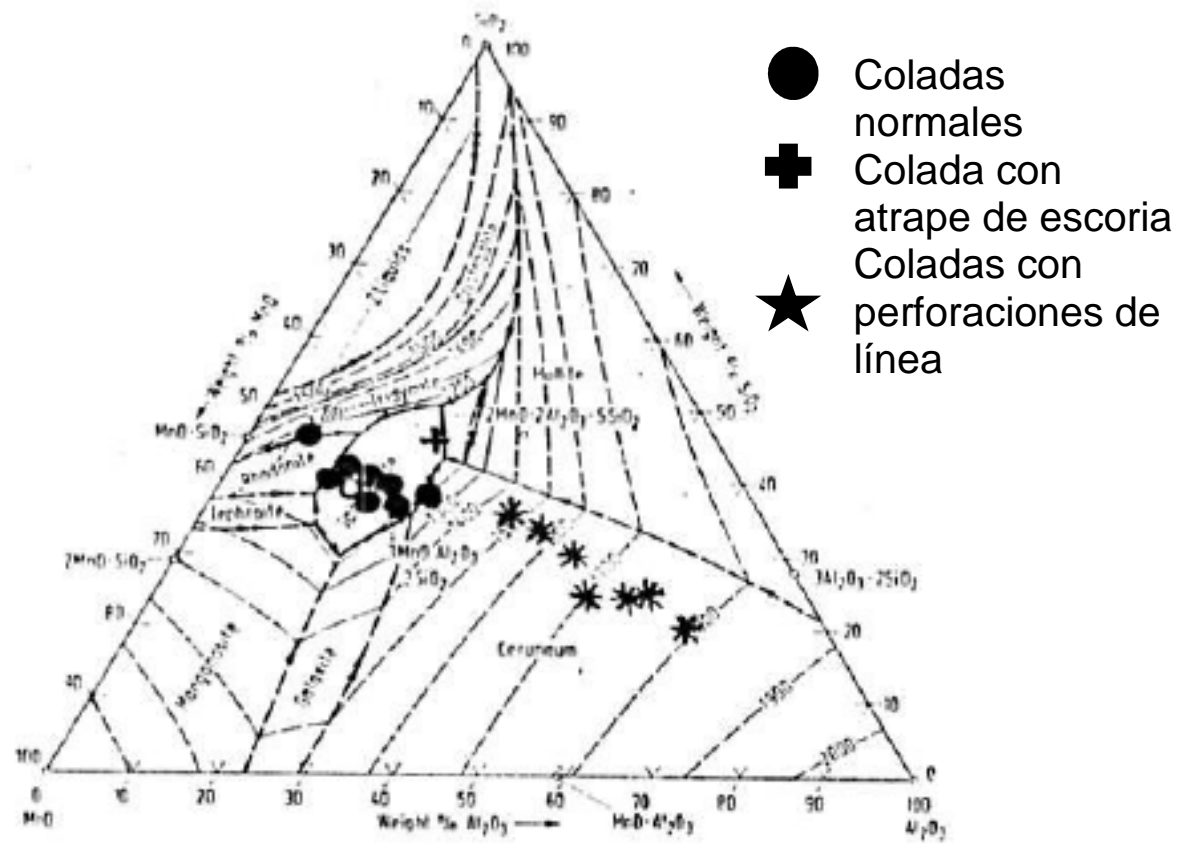

Figura 11. Ubicación en el diagrama de fase $\mathrm{MnO}-\mathrm{SiO} 2-\mathrm{Al} 2 \mathrm{O} 3$ de escorias de molde en colada continua de tochos a chorro abierto, con inyección de aluminio en el molde [12]. Coladas normales, con atrape de escoria y con perforaciones de línea.

\section{ANÁLISIS COMPARATIVO}

En la Tabla 1 se intenta una comparación cualitativa entre ambas prácticas, desde los puntos de vista de la seguridad y el ambiente laboral, el costo de inversión, el costo operativo, la productividad y la calidad de los productos.

* Contribuição técnica ao 45 Seminário de Aciaria - Internacional, 25 a 28 de maio de 2014, Porto Alegre, RS, Brasil. 
Tabla 1. Análisis comparativo entre dos estrategias de colado con buza calibrada

\begin{tabular}{|c|c|c|c|c|c|}
\hline & $\begin{array}{c}\text { Seguridad y } \\
\text { ambiente } \\
\text { laboral }\end{array}$ & $\begin{array}{l}\text { Costo de } \\
\text { inversión }\end{array}$ & $\begin{array}{c}\text { Costo } \\
\text { operativo }\end{array}$ & Productividad & Calidad \\
\hline $\begin{array}{l}\text { Chorro } \\
\text { abierto }\end{array}$ & $\begin{array}{c}\text { Salpicaduras } \\
\text { Formación de } \\
\text { chanchos en } \\
\text { repartidor que } \\
\text { requieren ser } \\
\text { cortados } \\
\text { Llamas de } \\
\text { combustión } \\
\text { del aceite } \\
\text { Exposición a } \\
\text { radiación } \\
\text { durante } \\
\text { "pesca" }\end{array}$ & $\begin{array}{l}\text { Sistema de } \\
\text { inyección de } \\
\text { aluminio } \\
\text { (optativo) }\end{array}$ & $\begin{array}{c}\text { Alambre de } \\
\text { aluminio } \\
\text { Mayor } \\
\text { consumo de } \\
\text { aceite } \\
\text { Necesidad de } \\
\text { personal por } \\
\text { línea } \\
\text { controlando } \\
\text { inyección de } \\
\text { aluminio y } \\
\text { "pescando" } \\
\text { escoria }\end{array}$ & $\begin{array}{c}\text { Similar } \\
\text { Se pueden } \\
\text { presentar } \\
\text { perforaciones } \\
\text { por escoria y } \\
\text { por colgadura }\end{array}$ & $\begin{array}{c}\text { Altas } \\
\text { macroinclusio } \\
\text { nes }\left(>20 / \mathrm{dm}^{2}\right) \\
\text { Atrapes de } \\
\text { escoria } \\
\text { Línea de lápiz } \\
\text { (aluminio en } \\
\text { la superficie } \\
\text { de la } \\
\text { palanquilla) }\end{array}$ \\
\hline $\begin{array}{c}\text { Tubo y } \\
\text { tambucho }\end{array}$ & $\begin{array}{c}\text { Puede haber } \\
\text { riesgo en la } \\
\text { colocación del } \\
\text { tubo, si el } \\
\text { sistema no es } \\
\text { adecuado, la } \\
\text { distancia es } \\
\text { corta, etc.; } \\
\text { puede haber } \\
\text { riesgo en la } \\
\text { limpieza del } \\
\text { tubo luego de } \\
\text { cada colada, } \\
\text { que implica el } \\
\text { uso de } \\
\text { oxígeno }\end{array}$ & $\begin{array}{c}\text { Costo de } \\
\text { sistema de } \\
\text { manipulació } \\
\mathrm{n} \text { de tubo y } \\
\text { de inyección } \\
\text { de argón por } \\
\text { el tope } \\
\text { Costo de } \\
\text { sistema de } \\
\text { control de } \\
\text { gas inerte y } \\
\text { de } \\
\text { tambucho/fu } \\
\text { elle }\end{array}$ & $\begin{array}{c}\text { Tubo } \\
\text { cerámico; } \\
\text { junta; argón } \\
\text { para tubo; } \\
\text { Argón o } \\
\text { nitrógeno para } \\
\text { tambucho } \\
\text { Menor } \\
\text { consumo de } \\
\text { aceite } \\
\text { No se utiliza la } \\
\text { inyección de } \\
\text { aluminio } \\
\text { Necesidad de } \\
\text { personal en los } \\
\text { arranques para } \\
\text { colocación del } \\
\text { tubo y } \\
\text { arranque de la } \\
\text { protección } \\
\text { gaseosa }\end{array}$ & Similar & $\begin{array}{c}\text { Menor } \\
\text { cantidad de } \\
\text { macroinclusio } \\
\text { nes }\left(<3 / \mathrm{dm}^{2}\right) \\
\text { Menor } \\
\text { posibilidad de } \\
\text { atrape de } \\
\text { nata } \\
\text { Menor } \\
\text { posibilidad de } \\
\text { perforaciones } \\
\text { No hay línea } \\
\text { de lápiz } \\
\text { Se pueden } \\
\text { colar aceros } \\
\text { con } \mathrm{Mn} / \mathrm{Si}<3\end{array}$ \\
\hline
\end{tabular}

En la comparación se puede observar que la práctica de protección $100 \%$ en el colado con buza calibrada implica un cierto costo de inversión y operativo, a cambio de una mayor seguridad del personal, un mejor ambiente laboral y una mejor calidad superficial e interna de los productos obtenidos. En cuanto a la mano de obra parecería tener más requerimientos en los arranques de secuencia y menos en la operación normal.

Es importante tener en cuenta que, desde el punto de vista de la optimización de una práctica, incluyendo la minimización de su costo operativo, no es lo mismo llevarla a cabo en unas coladas por mes (por ejemplo, las de bajo carbono) que hacerlo en el $100 \%$ de las coladas con buza calibrada.

\section{CONCLUSIONES}

Se plantean dos estrategias para el colado con buza calibrada y lubricación con aceite. La que algunas plantas han adoptado, haciendo el $100 \%$ de las coladas con buza calibrada con tubo de protección entre cuchara y repartidor y protección

* Contribuição técnica ao 45ำ Seminário de Aciaria - Internacional, 25 a 28 de maio de 2014, Porto Alegre, RS, Brasil. 
gaseosa entre el repartidor y el molde, implica incurrir en cierto costo de inversión y operativo pero proporcionaría más seguridad al personal, mejor ambiente laboral y mejor calidad superficial e interna de las palanquillas y por ende de los productos laminados.

\section{REFERENCIAS}

1 Palmaers A, Defays J, Philippe L. Deoxidation of continuously cast low carbon steel for billets. CRM Bulletin. 1979;55:16-23.

2 Mansell KP, Spencer DM, Vielhauer PA, Prestidge J, Varcoe DA. Record performance from steel delivery system refractories. In: 2000 Electric Furnace Conference Proceedings, November 12-15, Orlando, USA. ISS; 2000. p.477-491.

3 Youtube [página de la internet]. Auburn: Nucor Steel; 2010 [visitado en nov. 2013]. Disponible en: http://www.youtube.com/watch?v=tr3v-penL4s.

4 TradeKorea [página de la internet - visitado en nov. 2013]. Disponible en: http://www.tradekorea.com/e-catalogue/chosunref/productdetail/P00049630/Ladle_Shroud_Nozzle.html\#.UpNjnsRLMIQ.

5 TBR Casting Technologies [página de la internet]. Prettachstrasse:TBR [visitado en nov. 2013]. Disponible en: http://www.tbr-casting.com/products/continuouscasting/329-2/?lang=en.

6 Thomas B. Fluid flow in the mold. The Making, Shaping and Treating of the Steel, Chapter 14, The AISE Steel Foundation, 2003, pp. 1-41.

7 Tundish to mould bellow shroud systems for cleaner steel. CCR Technologies leaflet, en http://www.ccr-technologies.com/pdf/CCR-BROCHURE-BELLOWS\%20SHROUD.pdf, visitado en noviembre 2013.

8 Samarasekera IV, Brimacombe JK, Kumar S, Mclean A. The tundish pouring stream and cast billet quality. Proceedings Intl. Symp. on Near-Net-Shape Casting in the Minimills, Vancouver, Canada, 1995, pp. 135-160.

9 Maddever WJ, Mclean A, Luckett JS, Forward GE. An investigation of casting streams. Canadian Metallurgical Quarterly. 1973;12:79-88.

10 Farrell JW, Bilek PJ, Hilty DC. Inclusions originating from reoxidation of liquid steel. Electrice Furnace Proceedings, 1970, pp. 64-88.

11 Santillana B, Madías J, Dziuba M, Oropeza M, Fernández E. Eliminación de atrape de escoria y gotas frías en colada continua de palanquillas de acero. Revista de Metalurgia del CENIM. 2002;38:443-450.

12 Madías J, Garro M, Vénica R, Santachiara H, Castellá A, Lagos E. Formación y atrape de escoria en el molde en colada continua de tochos. Informe IAS - SOMISA Enero 1991.

* Contribuição técnica ao 45ํㅗㄴ Seminário de Aciaria - Internacional, 25 a 28 de maio de 2014, 